\title{
VARIATIONS OF FLOW DIRECTION IN SOLAR WIND STREAMS OF DIFFERENT TYPES
}

\author{
A.V. Moskaleva \\ Space Research Institute IKI RAS, \\ Moscow, Russia, mosanas6@yandex.ru \\ M.O. Riazantseva \\ Space Research Institute IKI RAS, \\ Moscow,Russia,orearm@gmail.com
}

\author{
Yu.I. Yermolaev \\ Space Research Institute IKI RAS, \\ Moscow,Russia,yermol@iki.rssi.ru \\ I.G. Lodkina \\ Space Research Institute IKI RAS, \\ Moscow, Russia, irina-priem@mail.ru
}

\begin{abstract}
Studying the direction of the solar wind flow is a topical problem of space weather forecasting. As a rule, the quiet and uniform solar wind propagates radially, but significant changes in the solar wind flow direction can be observed, for example, in compression regions before the interplanetary coronal mass ejections (Sheath) and Corotating Interaction Regions (CIR) that precede high-speed streams from coronal holes. In this study, we perform a statistical analysis of the longitude $(\varphi)$ and latitude $(\theta)$ flow direction angles and their variations on different time scales (30 s and $3600 \mathrm{~s}$ ) in solar wind large-scale streams of different types, using WIND spacecraft data. We also examine the relationships of the value and standard deviations SD of the flow direction angles with various solar wind parameters, regardless of the solar wind type.

We have established that maximum values of longitude and latitude angle modulus, as well as their variations, are observed for Sheath, CIR, and Rare, with the probability of large deviations from the radial direction
\end{abstract}

$\left(>5^{\circ}\right)$ increasing. The dependence on the solar wind type is shown to decrease with scale. We have also found that the probability of large values of $\operatorname{SD}(\theta)$ and $\operatorname{SD}(\varphi)$ increases with increasing proton temperature $\left(T_{\mathrm{p}}\right)$ in the range $5-10 \mathrm{eV}$ and with increasing proton velocity $\left(V_{\mathrm{p}}\right)$ in the range $400-500 \mathrm{~km} / \mathrm{s}$.

Keywords: solar wind, flow direction angles, types of solar wind.

\section{INTRODUCTION}

The undisturbed solar wind (SW) flow at a distance of $1 \mathrm{AU}$ is predominantly directed radially. When disturbed solar corona structures propagate in SW, streams interact, thereby causing the SW ion flux to deviate from the radial direction. A similar situation occurs, for example, in Corotating Interaction Regions (CIR) at boundaries of fast streams from coronal holes and slow streams from coronal streamers [Gosling, Pizzo, 1999]. Gosling and Pizzo [1999] have shown that the SW velocity vector deviates from westerly (negative longitude angle $\varphi$ ) to easterly (positive angle $\varphi$ ) direction, and this behavior of the $\varphi$ angle is explained by solar rotation and spatial distribution of the solar corona regions emitting SW streams with different velocities.

In [Yermolaev et al., 2015, 2018a ], the behavior of the longitude $\varphi$ and latitude $\theta$ angles of the velocity vector in large-scale SW streams of different types has been examined using hourly measurements from the OMNI database. It was confirmed that in CIR-type streams $\varphi$ increases on average from $-2^{\circ}$ to $+2^{\circ}$. In addition, it was shown that the longitude angle can change in a similar way in compression regions (Sheath) ahead of inter- planetary coronal mass ejections (ICME) of both types (Ejecta) or magnetic clouds (MC), the $\varphi$ angle changing in the opposite direction (from $+2^{\circ}$ to $-2^{\circ}$ ) inside Ejecta and MC.

The mean change in the latitude angle $\theta$ was demonstrated to be almost constant, about one degree for all SW types. The number of events with a tendency for $\theta$ to increase or decrease does not exceed $15 \%$ [Yermolaev et al., 2018a]. If such a change takes place in CIR and Sheath, the $\theta$ angle changes similarly to the $\varphi$ angle: the absolute value of the angle increases, reaches a maximum, decreases, and changes sign to the opposite one which was at the beginning of the interval.

The aforementioned strong changes in the flux vector direction during the collision of large-scale SW streams occur on time scales of $\geq 1 \mathrm{hr}\left(\geq 10^{6} \mathrm{~km}\right)$. Significant changes in the flow direction can also occur in SW plasma at the boundaries of flux tubes with scales of $\sim(4-5) \cdot 10^{5} \mathrm{~km}$ [Borovsky, 2008].

Such processes can be described by the MHD approximation. At the same time, on spatial scales $10^{3}-10^{5}$ $\mathrm{km}$ there may by short-term and significant deviations of the flux vector due to local processes in SW plasma. 
For example, Zastenker et al. [2015] have shown that for most of the observation time fast variations in the polar angle are within $0.2^{\circ}-0.5^{\circ}$, but sometimes (on average, several times a day) there are fast jumps in the polar angle by $5^{\circ}-10^{\circ}$ and higher. Such jumps are unevenly distributed over time and can be associated with the "jet" nature of the SW flow.

Studying the direction of the flux vector and its variations is a topical problem for a more accurate understanding of the processes of SW formation and its dynamics. The deviation of the flux vector from the radial direction can also be used for predicting space weather, since it allows one to identify CIR and Sheath, which are highly geoeffective [Yermolaev et al., 2018b].

This paper statistically analyzes angles of SW flux vector direction and their variations for various largescale SW types from WIND spacecraft data. We examine the averaging intervals of 30 and $3600 \mathrm{~s}$. Most of the previous works (e.g., [Yermolaev et al., 2015, 2018a; Gosling, Pizzo, 1999; Borovsky, 2008]) have described large-scale or medium-scale variations in the flow direction angles, whereas papers dealing with small-scale variations (e.g., [Zastenker et al., 2015]) used limited statistical material. In this paper, for the first time we perform a systematic statistical analysis of variations in flow angles on scales $\sim 10^{3}-10^{5} \mathrm{~km}$, using long-term measurements. We also analyze the dependences of values and standard deviations of flow direction angles on different SW parameters, regardless of its type.

\section{DATA AND ANALYSIS METHOD}

We study values and variations of the flow direction angles, using WIND spacecraft measurements of SW parameters for the period 1995-2019, presented in the database [http://cdaweb.gsfc.nasa.gov]. We have used data on SW proton velocity, temperature, and density from a set of PM measurements of the 3DP instrument [Lin et al., 1995], as well as data on the magnitude and components of the interplanetary magnetic field (IMF) from a set of $\mathrm{H} 0$ measurements of the MFI instrument [Lepping et al., 1995]. Time resolution of the parameters analyzed is $\sim 3 \mathrm{~s}$.

All data we employ is in the geocentric solar-ecliptic (GSE) coordinate system: the $\mathrm{X}$-axis is directed from Earth to the Sun, the Y-axis is located in the ecliptic plane and is directed opposite to the planetary motion, the Z-axis is the normal to the ecliptic plane and complements the right-hand coordinate system (northward). In the datasets in use, the flow direction angles were not given separately, and their values were calculated from proton velocity components, using the following formulas: $V_{x}=-V \cos (\theta) \cos (\varphi) ; \quad V_{y}=V \cos (\theta) \sin (\varphi) ; \quad V_{z}=V \sin (\theta)$, where $V_{x}, V_{y}, V_{z}$ are the $\mathrm{SW}$ proton velocity components; $V$ is the SW proton velocity modulus; $\theta$ and $\varphi$ are the latitude and longitude angles respectively.

To study variations in angles on different scales, we have divided time series into 30 and $3600 \mathrm{~s}$ intervals. We chose the duration of the intervals to cover a wide range of variation scales. At the same time, we did not aim at studying large-scale variations repeatedly described in earlier works (see Introduction), which determined the choice of the maximum interval duration. The minimum duration of the interval was determined by representativeness of sample, taking into account time resolution of the data in hand. Each subsequent interval overlapped the previous one by half its length, and if any abrupt change in the angles at the boundary of streams occurred at the junction of the intervals and was not included into the statistics, then, in examining the next interval, this change was in its center and could be reliably considered. For each data interval, we calculated averaged values of the longitude and latitude angles and mean values of the remaining SW and IMF parameters. To analyze variations in the flow direction angles, at each interval we calculated values of their sample standard deviation SD. In addition, each interval was referred to a certain SW type according to the catalog of large-scale SW phenomena [Yermolaev et al., 2009; http://www.iki. rssi.ru/omni/catalog]. In the sample considered, we identified the following SW types:

1. Ejecta - interplanetary coronal mass ejections (ICMEs). The duration averages around $30 \mathrm{hrs}$.

2. MC (Magnetic Cloud) - magnetic clouds, which, like Ejecta, are generated by CMEs, but, unlike Ejecta, have a higher and more regular (rotating in most cases) IMF. MC has an average duration of $28 \mathrm{hrs}$.

3. CIR (Corotating Interaction Region) - compression regions in front of the incoming fast stream from coronal holes. Duration of the SW stream of this type is on average $20 \mathrm{hrs}$.

4. Sheath - compression regions ahead of fast Ejecta/MC. The duration of Sheath in SW is on average 16 hrs. CIR and Sheath are similar in parameters and differ only in the type of piston compressing the plasma.

5. Rare - a rarefaction region, plasma between the fast SW stream that moves away from the slow one. Duration of the rarefied region averages around 10-20 hrs.

6. HCS - the heliospheric current sheet. This type is related to the SW sector structure. The HCS duration is $\sim 5 \mathrm{hrs}$.

7. Fast - fast quasi-stationary SW associated with coronal holes.

8. Slow - slow quasi-stationary SW associated with coronal streamers.

The catalog of large-scale SW phenomena [Yermolaev et al., 2009] describes in detail the criteria and methods for identifying SW stream types.

Table 1 shows the number of intervals obtained for each large-scale SW stream type for a $30 \mathrm{~s}$ averaging interval as a percentage of all intervals considered. We can see that Rare is the rarest of the SW stream types under study. Note that the duration of the intervals considered is much shorter than the mean duration of observation of the above large-scale SW stream types, and only one SW type is observed in each of the intervals. The intervals in which two or more SW stream types were observed simultaneously were excluded during selection.

The WIND spacecraft is located at the libration point L1, which is $\sim 1.5$ million $\mathrm{km}$ from Earth; therefore, to refer 
it to the catalog of large-scale SW phenomena, we took into account the time of SW propagation to Earth's orbit.

For the 30 and $3600 \mathrm{~s}$ averaging intervals, we carried out a statistical analysis of absolute values of the longitude and latitude flow direction angles and their variations. In addition, we examined the dependences of the value and standard deviations of the flow direction angles on various SW and IMF parameters.

\section{RESULTS}

\subsection{Statistical analysis of values and varia- tions of SW flow direction angles}

Below are the results of the statistical analysis of the absolute values of the longitude and latitude angles $(|\varphi|$ and $|\theta|$ respectively) and their standard deviations for various large-scale SW stream types. We also compare the results obtained for fast, $30 \mathrm{~s}$, and slow, $3600 \mathrm{~s}$, variations.

Figure 1 illustrates the distributions of the average latitude $|\theta|$ and longitude $|\varphi|$ angles calculated for $30 \mathrm{~s}$ intervals. Figure 1, $a, c$ shows the distributions for the intervals selected for HCS, MC, Ejecta, and Slow; and
Figure 1, $b$ and $d$, for Slow, Fast, Rare, Sheath, and CIR. Positions of the distribution maxima are the same for all SW types, except for the distribution of the longitude angle for $\mathrm{MC}$; however, the distribution width and mean values explicitly depend on the large-scale stream type. The average absolute values of the angles and positions of maxima of their distributions are listed in Table 4. For CIR, Sheath, MC, and Rare, the distributions are wider than for HCS, Slow, Fast, and Ejecta. Similar histograms of absolute values of the angles were also plotted for $3600 \mathrm{~s}$ intervals, but they did not exhibit significant differences from the histograms for $30 \mathrm{~s}$ intervals.

Note that the histograms in Figure 1 also show a noticeable number of intervals with the latitude and longitude angles exceeding $5^{\circ}$ in modulus. Table 2 lists the probabilities of observing absolute values of $>5^{\circ}$ angles for different SW types. For CIR, Sheath, MC, and Rare, the $>5^{\circ}$ deviations occur more often than for other SW stream types, the maximum number of such intervals being observed for Rare.

Table 1

The number of intervals obtained for each large-scale SW stream type with a $30 \mathrm{~s}$ averaging interval as a percentage of all intervals considered

\begin{tabular}{|c|c|c|c|c|c|c|c|}
\hline HCS & MC & EJECTA & SLOW & FAST & RARE & SHEATH & CIR \\
\hline $6.9 \%$ & $1.6 \%$ & $10.5 \%$ & $43.8 \%$ & $26.1 \%$ & $0.6 \%$ & $3.2 \%$ & $7.3 \%$ \\
\hline
\end{tabular}

a)

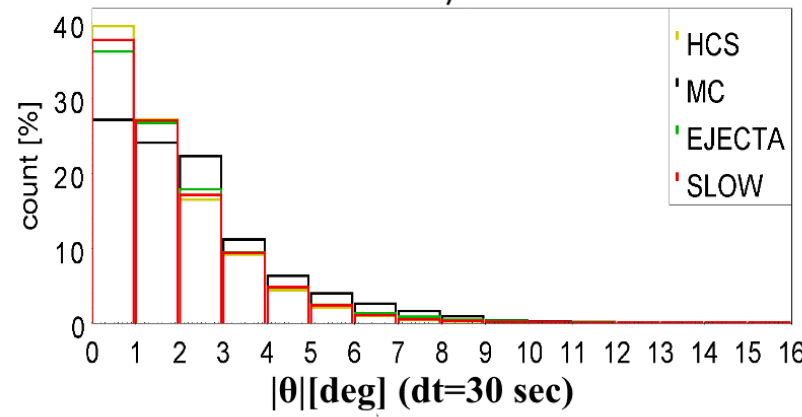

b)

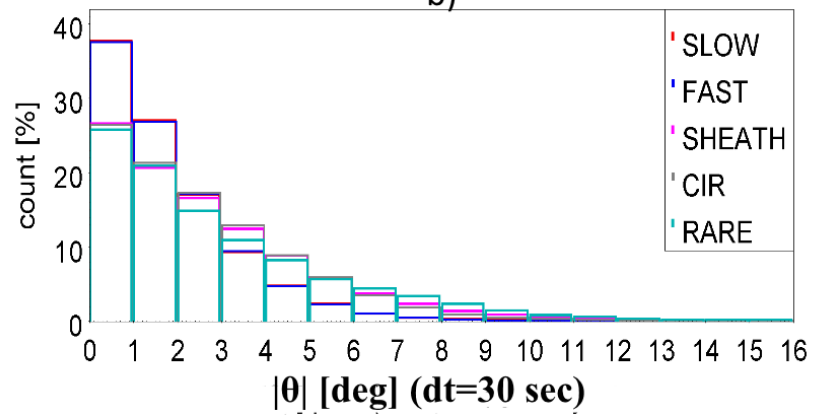

c)

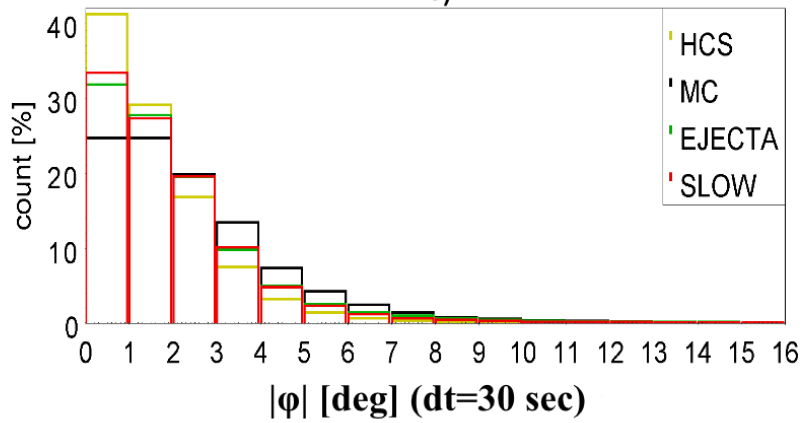

d)

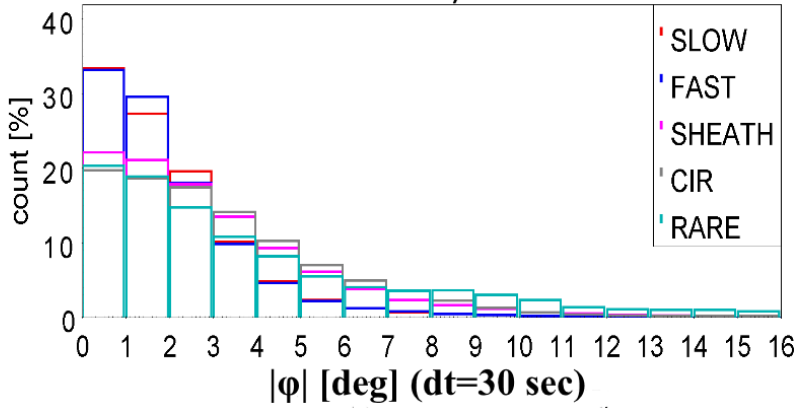

Figure 1. Distribution of average latitude $|\theta|$ and longitude $|\varphi|$ angles over $30 \mathrm{~s}$ intervals

Table 2

Observation probability of absolute values of latitude $\theta$ and longitude $\varphi$ angles of $>5^{\circ}$ for different SW types

\begin{tabular}{|c|c|c|c|c|c|c|c|c|}
\hline & HCS & MC & EJECTA & SLOW & FAST & RARE & SHEATH & CIR \\
\hline$|\theta|$ & $4 \%$ & $9 \%$ & $5 \%$ & $4 \%$ & $4 \%$ & $20 \%$ & $15 \%$ & $13 \%$ \\
\hline$|\varphi|$ & $2 \%$ & $11 \%$ & $6 \%$ & $5 \%$ & $5 \%$ & $27 \%$ & $16 \%$ & $20 \%$ \\
\hline
\end{tabular}


To study the level of variations in the flow direction angles in different SW stream types, we have plotted similar distributions for standard deviations of the latitude $\operatorname{SD}(\theta)$ and longitude $\operatorname{SD}(\varphi)$ angles. Figure 2 presents the distributions of standard deviations of both angles for $30 \mathrm{~s}$ intervals; and Figure 3, for $3600 \mathrm{~s}$ intervals. Figures 2, $a, c$ and 3, $a, c$ show the distributions for HCS, MC, Ejecta, and Slow; Figures 2, $b, d$ and 3, $b, d$, for Slow, Fast, Rare, Sheath, and CIR. Maxima of the distributions for $30 \mathrm{~s}$ intervals are the same for all SW types, except for Rare; the distribution width and mean values explicitly depend on the large-scale stream type. For 3600 s intervals, significant differences are observed in particular for positions of the distribution maxima. Positions of maxima and average SD distributions for both averaging intervals

a)

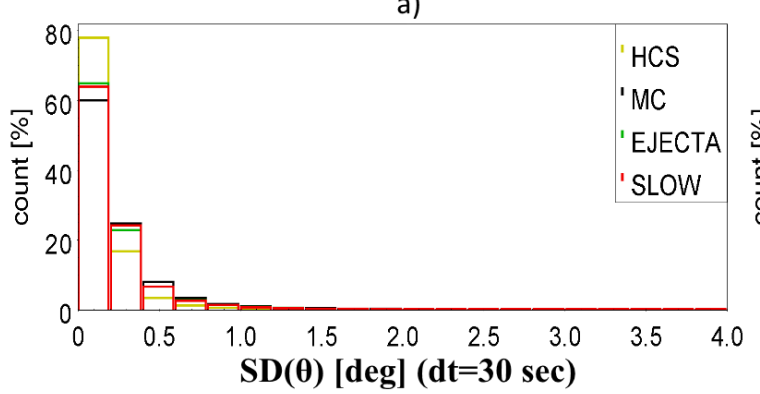

b)

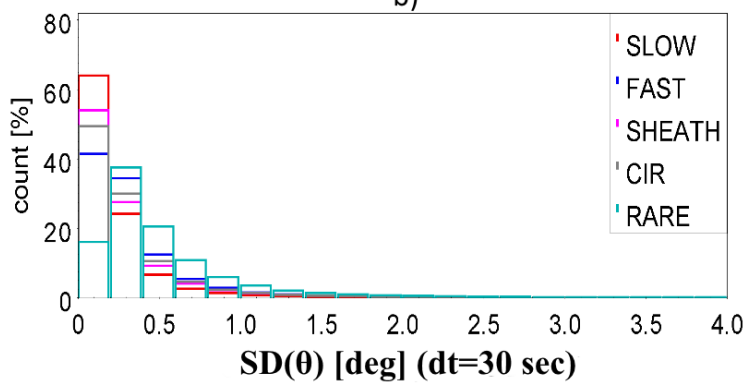

are presented in Table 4. The SD distributions are wider for CIR, Sheath, Fast, and Rare than for HCS, Slow, Ejecta, and MC.

Note that the SD value characterizing the level of variations in the latitude and longitude angles strongly depends on the length of the interval: with decreasing time scale, both the amplitude of the variations and the probability of occurrence of large SD values decrease. This is due to the fact that strong changes in direction angles generally occur relatively slowly - for about tens of minutes. Sharp and large-scale changes in flow direction with periods of the order of seconds occur quite rarely.

c)

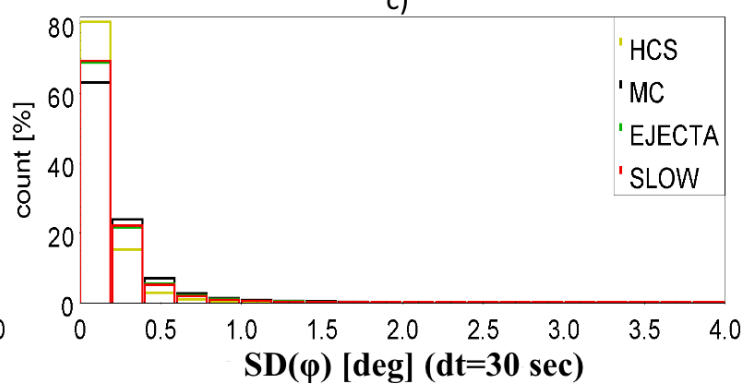

d)

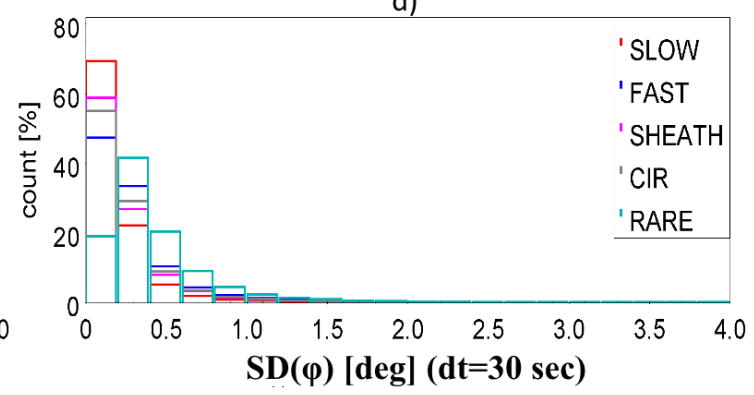

Figure 2. Distributions of standard deviations of latitude $\operatorname{SD}(\theta)$ and longitude $\operatorname{SD}(\varphi)$ angles over 30 s intervals

a)

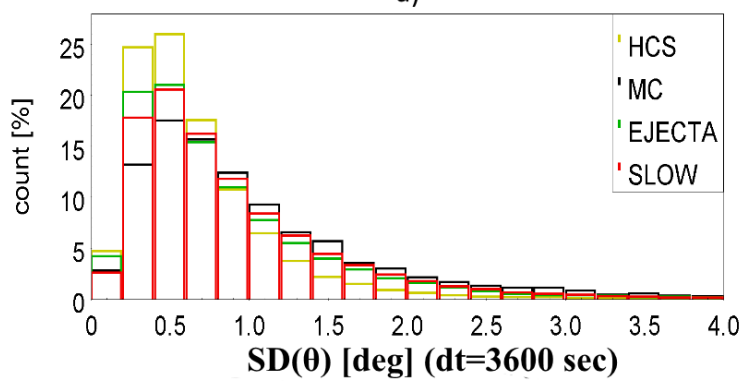

c)

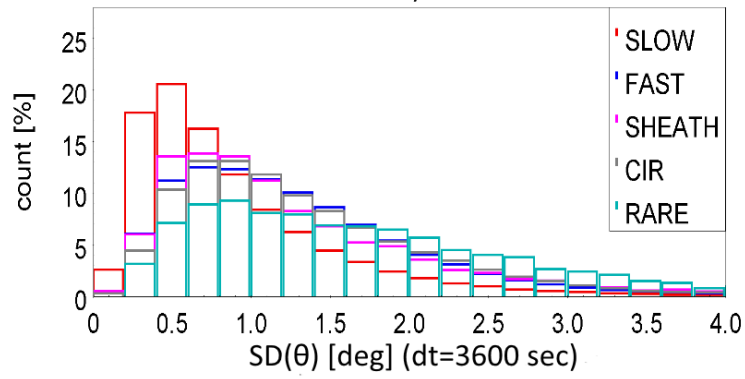

b)

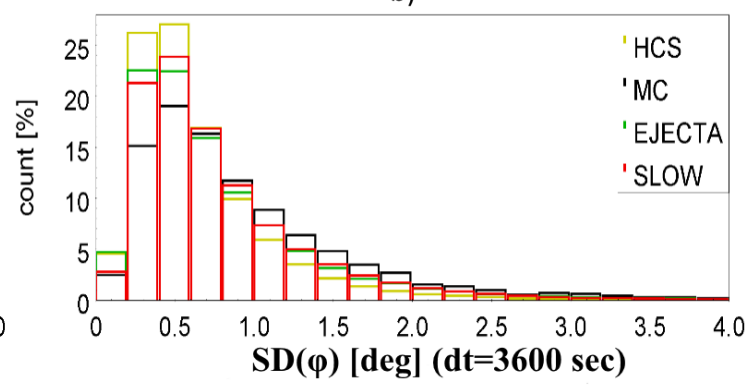

d)

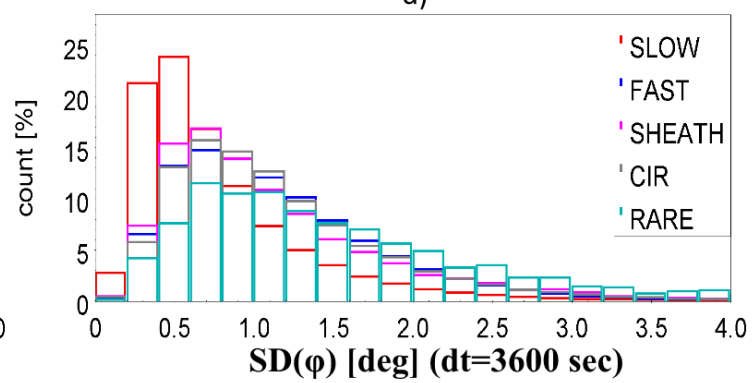

Figure 3. Distributions of standard deviations of latitude $\operatorname{SD}(\theta)$ and longitude $\operatorname{SD}(\varphi)$ angles over $3600 \mathrm{~s}$ intervals 
Thus, the SD distributions over long time intervals (3600 s) generally reflect the variations associated with medium-scale changes in the SW flow direction, for example, between flux tubes [Borovsky, 2008; 2018], whereas the SD distributions over short intervals (30 s) reflect the level of local fluctuations in the flow direction linked to the small-scale structure of the SW flow.

The histograms of standard deviations of the angles for slow $3600 \mathrm{~s}$ variations show a noticeable number of intervals with standard deviations of the latitude and longitude angles exceeding $2^{\circ}$. Table 3 presents the probabilities of observing $>2^{\circ}$ variations for different SW types at $3600 \mathrm{~s}$ intervals. The probability of large deviations for both angles increases for CIR, Sheath, Fast, MC, and Rare, noticeably exceeding the similar probability for other SW stream types.

A summary characteristic for all the aforementioned distributions is given in Table 4. It lists averages $(<|\theta|>$, $\langle|\varphi|\rangle, \quad\langle\mathrm{SD}(\theta)\rangle_{30 \mathrm{~s}}, \quad\langle\mathrm{SD}(\varphi)\rangle_{30 \mathrm{~s}}, \quad\langle\mathrm{SD}(\theta)\rangle_{3600 \mathrm{~s}}$, $\left.\langle\mathrm{SD}(\varphi)\rangle_{3600 \mathrm{~s}}\right)$ and maxima $\max (|\theta|), \quad \max (|\varphi|)$, $\max (\operatorname{SD}(\theta))_{30 \mathrm{~s}}, \quad \max (\operatorname{SD}(\varphi))_{30 \mathrm{~s}}, \quad \max (\operatorname{SD}(\theta))_{3600 \mathrm{~s}}$, $\max (\operatorname{SD}(\varphi))_{3600 \mathrm{~s}}$ of absolute values of angles and standard deviations (for both averaging intervals) for all SW stream types.

\subsection{Study of dependences of flow direction angles and their variations on SW parameters}

Besides the statistical analysis of the values and variations of the flow direction angles for different time scales, we have studied the dependences of the angles and their standard deviations on various SW and IMF plasma parameters. Note that this Section deals with the entire set of measurements without dividing into largescale SW types. Below are similar dependences for those SW parameters for which there is a clear relationship of the angles and their standard deviations with the respective SW parameter.

Figure 4 plots the latitude angle $\theta$ (Figure $4, a$ ) and the longitude angle $\varphi$ (Figure $4, c$ ) as a function of proton velocity $V_{\mathrm{p}}$. Color indicates the number of intervals in the selected range of parameter values. Velocities from 250 to $800 \mathrm{~km} / \mathrm{s}$ are plotted along the $\mathrm{X}$-axis and are divided into ranges with a step of $10 \mathrm{~km} / \mathrm{s}$; the $\theta$ and $\varphi$ angles from $-10^{\circ}$ to $+10^{\circ}$ are along the $\mathrm{Y}$-axis and divided into ranges with a step of $1^{\circ}$. The color scale on the right indicates the correspondence of the color to the number of points in each cell. For the latitude angle $\theta$ (Figure $4, a$ ), a symmetric distribution is observed regardless of the velocity, with the distribution maximum slightly shifted toward positive values $\left(\sim+1^{\circ}\right)$.

For the longitude angle $\varphi$ (Figure $4, c$ ), there is a clear velocity dependence of the distribution shape. For low velocities $250-450 \mathrm{~km} / \mathrm{s}$, there is a symmetric distribution with a maximum shifted toward negative values $\left(\sim-2^{\circ}\right)$. The proportion of the intervals with $\varphi \geq 0^{\circ}$ and $\varphi<0^{\circ}$ is 36.5 and $63.5 \%$ respectively. At $>450 \mathrm{~km} / \mathrm{s}$ velocities, the distribution becomes asymmetric, the probability of positive $\varphi$ values increases with velocity: the proportion of intervals with $\varphi \geq 0^{\circ}$ is $47.7 \%$; and with $\varphi<0^{\circ}, 52.3 \%$; i.e., the number of intervals with negative and positive values of the longitude angle becomes almost the same, whereas at low velocities the proportion of intervals with negative values of the longitude angle is noticeably larger.

Figure 4, $b$ and $d$ show similar $\theta$ and $\varphi$ dependences on proton temperature $T_{\mathrm{p}}$. The $T_{\mathrm{p}}$ values plotted along the $\mathrm{X}$-axis from 2 to $50 \mathrm{eV}$ are divided into ranges with a step of $1 \mathrm{eV}$.

Note that the $V_{\mathrm{p}}$ and $T_{\mathrm{p}}$ values averaged over long periods of time demonstrate a positive correlation [Lopez, 1987]. However, the velocity and temperature ratios differ for different SW stream types and therefore are used for their identification [Yermolaev et al., 2009]. Despite the similarity between images for velocity and temperature, Figure $4, b$ and $d$ provides additional information about the relationship of the latitude and longitude angles with SW parameters. More detailed data on the relationship of the $\theta$ and $\varphi$ angles with interplanetary parameters in different SW types is planned to be presented in subsequent publications.

Probability of observing variations in latitude $\operatorname{SD}(\theta)$ and longitude $\operatorname{SD}(\varphi)$ angles of $>2^{\circ}$

Table 3 for different $\mathrm{SW}$ types $(3600 \mathrm{~s})$

\begin{tabular}{|c|c|c|c|c|c|c|c|c|}
\hline & HCS & MC & EJECTA & SLOW & FAST & RARE & SHEATH & CIR \\
\hline $\operatorname{SD}(\theta)$ & $2 \%$ & $11 \%$ & $6 \%$ & $7 \%$ & $16 \%$ & $36 \%$ & $17 \%$ & $17 \%$ \\
\hline $\operatorname{SD}(\varphi)$ & $2 \%$ & $10 \%$ & $5 \%$ & $4 \%$ & $11 \%$ & $27 \%$ & $12 \%$ & $11 \%$ \\
\hline
\end{tabular}

Maxima and averages of distributions of absolute values of longitude and latitude angles

Table 4 and their standard deviations (for $30 \mathrm{~s}$ and $3600 \mathrm{~s}$ averaging intervals)

\begin{tabular}{|l|c|c|c|c|c|c|}
\hline & $\langle|\theta|>,\langle|\varphi|\rangle$ & $\begin{array}{c}\langle\mathrm{SD}(\theta)\rangle_{30}, \\
\langle\mathrm{SD}(\varphi)\rangle_{30 \mathrm{c}}\end{array}$ & $\begin{array}{c}\langle\mathrm{SD}(\theta)\rangle_{3600 \mathrm{c}}, \\
\langle\mathrm{SD}(\varphi)\rangle_{3600 \mathrm{c}}\end{array}$ & $\begin{array}{c}\max (|\theta|), \\
\max (|\varphi|)\end{array}$ & $\begin{array}{c}\max (\mathrm{SD}(\theta))_{30 c}, \\
\max (\mathrm{SD}(\varphi))_{30 \mathrm{c}}\end{array}$ & $\begin{array}{c}\max (\mathrm{SD}(\theta))_{3600 \mathrm{c}}, \\
\max \left(\mathrm{SD}_{(}(\varphi)\right)_{3600 \mathrm{c}}\end{array}$ \\
\hline HCS & $1.71^{\circ}, 1.59^{\circ}$ & $0.15^{\circ}, 0.15^{\circ}$ & $0.66^{\circ}, 0.65^{\circ}$ & $0^{\circ}-1^{\circ}, 0^{\circ}-1^{\circ}$ & $0^{\circ}-0.2^{\circ}, 0^{\circ}-0.2^{\circ}$ & $0.4^{\circ}-0.6^{\circ}, 0.4^{\circ}-0.6^{\circ}$ \\
\hline MC & $2.32^{\circ}, 2.50^{\circ}$ & $0.24^{\circ}, 0.23^{\circ}$ & $1.00^{\circ}, 0.92^{\circ}$ & $0^{\circ}-1^{\circ}, 1^{\circ}-2^{\circ}$ & $0^{\circ}-0.2^{\circ}, 0^{\circ}-0.2^{\circ}$ & $0.4^{\circ}-0.6^{\circ}, 0.4^{\circ}-0.6^{\circ}$ \\
\hline EJECTA & $1.89^{\circ}, 2.07^{\circ}$ & $0.22^{\circ}, 0.20^{\circ}$ & $0.82^{\circ}, 0.76^{\circ}$ & $0^{\circ}-1^{\circ}, 0^{\circ}-1^{\circ}$ & $0^{\circ}-0.2^{\circ}, 0^{\circ}-0.2^{\circ}$ & $0.4^{\circ}-0.6^{\circ}, 0.2^{\circ}-0.4^{\circ}$ \\
\hline SLOW & $1.79^{\circ}, 1.96^{\circ}$ & $0.22^{\circ}, 0.20^{\circ}$ & $0.88^{\circ}, 0.77^{\circ}$ & $0^{\circ}-1^{\circ}, 0^{\circ}-1^{\circ}$ & $0^{\circ}-0.2^{\circ}, 0^{\circ}-0.2^{\circ}$ & $0.4^{\circ}-0.6^{\circ}, 0.4^{\circ}-0.6^{\circ}$ \\
\hline FAST & $1.80^{\circ}, 1.94^{\circ}$ & $0.33^{\circ}, 0.29^{\circ}$ & $1.26^{\circ}, 1.14^{\circ}$ & $0^{\circ}-1^{\circ}, 0^{\circ}-1^{\circ}$ & $0^{\circ}-0.2^{\circ}, 0^{\circ}-0.2^{\circ}$ & $0.6^{\circ}-0.8^{\circ}, 0.6^{\circ}-0.8^{\circ}$ \\
\hline RARE & $3.01^{\circ}, 3.91^{\circ}$ & $0.50^{\circ}, 0.44^{\circ}$ & $1.61^{\circ}, 1.45^{\circ}$ & $0^{\circ}-1^{\circ}, 0^{\circ}-1^{\circ}$ & $0.2^{\circ}-0.4^{\circ}, 0.2^{\circ}-0.4^{\circ}$ & $0.8^{\circ}-1.0^{\circ}, 0.6^{\circ}-0.8^{\circ}$ \\
\hline SHEATH & $2.70^{\circ}, 2.94^{\circ}$ & $0.28^{\circ}, 0.24^{\circ}$ & $1.23^{\circ}, 1.11^{\circ}$ & $0^{\circ}-1^{\circ}, 0^{\circ}-1^{\circ}$ & $0^{\circ}-0.2^{\circ}, 0^{\circ}-0.2^{\circ}$ & $0.6^{\circ}-0.8^{\circ}, 0.6^{\circ}-0.8^{\circ}$ \\
\hline CIR & $2.59^{\circ}, 3.19^{\circ}$ & $0.29^{\circ}, 0.26^{\circ}$ & $1.30^{\circ}, 1.14^{\circ}$ & $0^{\circ}-1^{\circ}, 0^{\circ}-1^{\circ}$ & $0^{\circ}-0.2^{\circ}, 0^{\circ}-0.2^{\circ}$ & $0.6^{\circ}-1.0^{\circ}, 0.6^{\circ}-0.8^{\circ}$ \\
\hline
\end{tabular}


a)

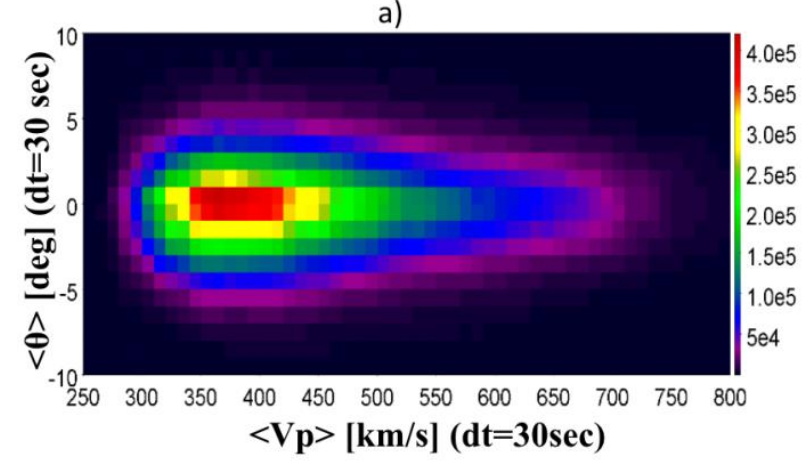

b)

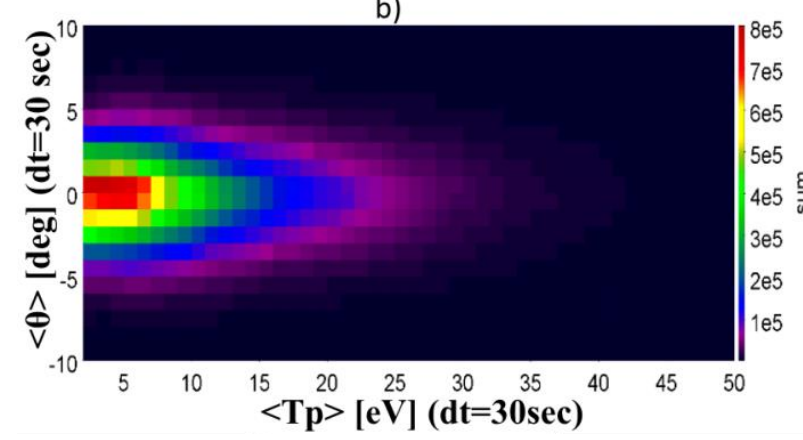

c)

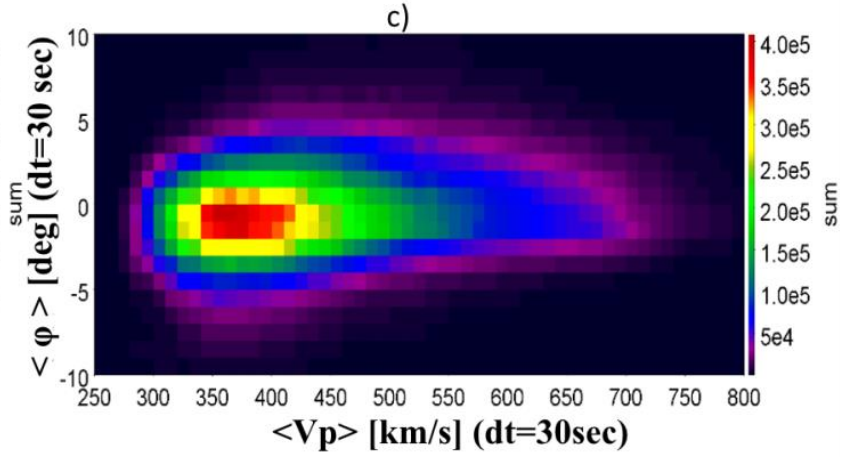

d)

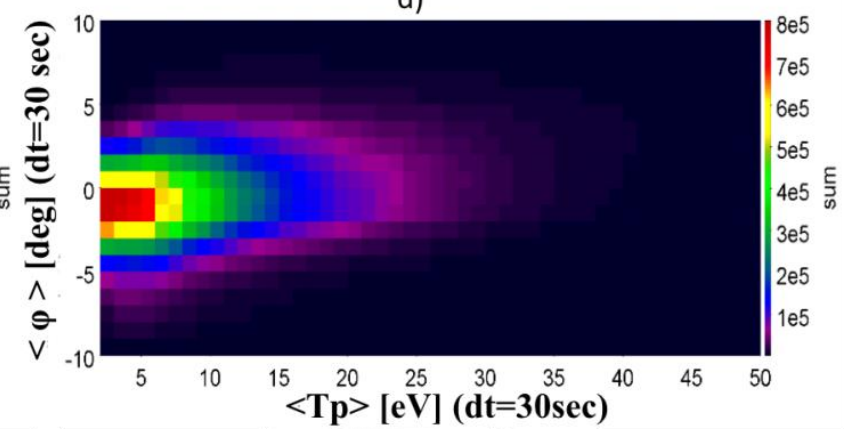

Figure 4. Latitude $\theta$ and longitude $\varphi$ angles as a function of proton velocity $V_{\mathrm{p}}$ and temperature $T_{\mathrm{p}}$

For the $\theta$ angle (Figure $4, b$ ), a symmetric distribution is also observed regardless of proton temperature, and for the $\varphi$ angle (Figure 4, $d$ ) the distribution is asymmetric and depends on $T_{\mathrm{p}}$. For low temperatures from 2 to $5 \mathrm{eV}$, the $\varphi$ angle distribution shifts toward negative values: proportions of the intervals with $\varphi \geq 0^{\circ}$ and $\varphi<0^{\circ}$ are 28.4 and $71.6 \%$ respectively. As in the case of velocity, the probability of positive $\varphi$ increases with $T_{\mathrm{p}}$. In the case of high temperatures (above $10 \mathrm{eV}$ ), the $\varphi$ angle distribution shifts toward positive values; proportions of the intervals with $\varphi \geq 0^{\circ}$ and $\varphi<0^{\circ}$ are approximately equal and amount to 51.7 and $48.3 \%$ respectively.

Figure 4 plots averaged $\theta$ and $\varphi$ angles as a function of the mean velocity and temperature, calculated for the $30 \mathrm{~s}$ intervals. The $3600 \mathrm{~s}$ intervals exhibit similar dependences (therefore omitted).

Figure 5 shows standard deviations of the latitude $\operatorname{SD}(\theta)$ (Figure 5, a) and longitude $\operatorname{SD}(\varphi)$ (Figure 5,c) angles as a function of $V_{\mathrm{p}}$ for $30 \mathrm{~s}$ intervals. Color indicates the number of intervals in the selected range of parameter values. Velocities from 250 to $800 \mathrm{~km} / \mathrm{s}$ are plotted along the $\mathrm{X}$-axis and are divided into ranges with a step of $10 \mathrm{~km} / \mathrm{s}$; the $\operatorname{SD}(\theta)$ and $\operatorname{SD}(\varphi)$ variations from $0^{\circ}$ to $1^{\circ}$ are plotted along the Y-axis and are divided into ranges with a step of $0.05^{\circ}$. The color scale on the right indicates the correspondence of the color to the number of points in each cell. Both dependences are similar. The probability of occurrence of large values increases with an increase in the velocity from 400-500 $\mathrm{km} / \mathrm{s}$; there is also a weak dependence of the position of the maximum on the velocity in the interval of 320 to $420 \mathrm{~km} / \mathrm{s}$.

Similar dependences of $\operatorname{SD}(\theta)$ and $\operatorname{SD}(\varphi)$ on $T_{\mathrm{p}}$ are presented in Figure 5, $b$ and $d$. The $T_{\mathrm{p}}$ values from 2 to $50 \mathrm{eV}$ are plotted along the $\mathrm{X}$-axis and are divided into ranges with a step of $1 \mathrm{eV}$. As in the case of the velocity dependences, the proton temperature dependences of standard deviations of the latitude and longitude angles appear similar. The probability of occurrence of large $\operatorname{SD}(\theta)$ and $\mathrm{SD}(\varphi)$ values increases when $T_{\mathrm{p}}$ exceeds 5-10 eV. There is a weak dependence of the position of the maximum on temperature in the range $2-7 \mathrm{eV}$.

Above we have presented the dependences of averaged standard deviations of the $\theta$ and $\varphi$ angles on average velocity and temperature for $30 \mathrm{~s}$ intervals. For $3600 \mathrm{~s}$ intervals, similar dependences are observed (not shown here), but the amplitudes of $\operatorname{SD}(\theta)$ and $\operatorname{SD}(\varphi)$ are $\sim 4$ times higher.

We have also plotted the latitude and longitude angles and their standard deviations as a function of proton density and IMF magnitude (omitted), but found no features, as in the case of the dependences on velocity and temperature.

\section{DISCUSSION AND CONCLUSIONS}

We have carried out a statistical analysis of the absolute values of longitude $\varphi$ and latitude $\theta$ SW flow direction angles and their variations on time scales of 30 and $3600 \mathrm{~s}$ for different large-scale SW stream types, using WIND data for the period 1995-2019. In addition, we have plotted and analyzed dependences of these values on different SW parameters, without dividing them into stream types.

As a result of the analysis:

1 . We have confirmed that the longitude angle $|\varphi|$ associated with the solar rotation is always greater than the latitude angle $|\theta|$, and the absolute values of both angles depend on the large-scale SW stream type [Yermolaev et al, 2018a]. Absolute values of the angles take the minimum values for HCS, Slow, Fast, Ejecta: $|\theta| \approx 1.7^{\circ}-1.9^{\circ}$ and $|\varphi| \approx 1.6^{\circ}-2.1^{\circ}$, and increase for CIR, 
a)

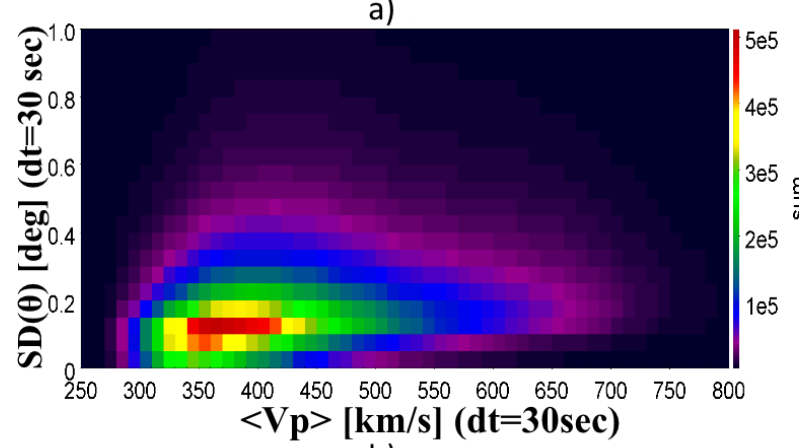

b)

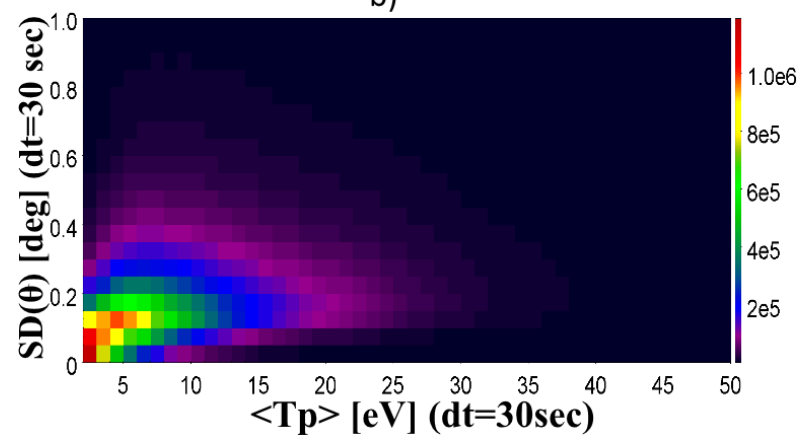

c)

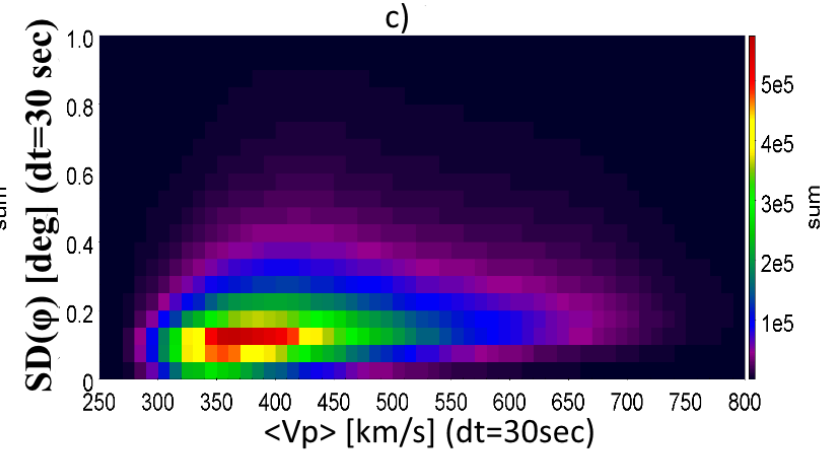

d)

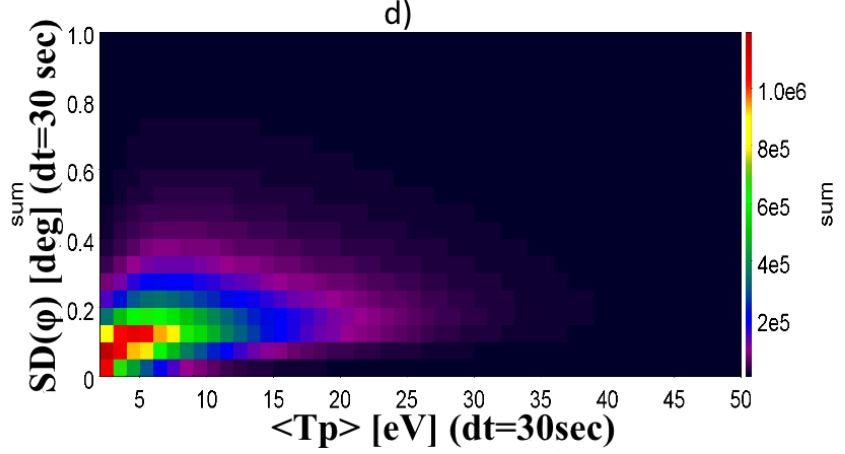
ture $T_{\mathrm{p}}$

Figure 5. Standard deviations of latitude $\operatorname{SD}(\theta)$ and longitude $\operatorname{SD}(\varphi)$ angles as a function of proton velocity $V_{\mathrm{p}}$ and tempera-

Sheath, MC: $|\theta| \approx 2.3^{\circ}-2.7^{\circ}$ and $|\varphi| \approx 2.5^{\circ}-3.2^{\circ}$. Maximum values are observed for Rare: $|\theta| \approx 3^{\circ}$ and $|\varphi| \approx 3.9^{\circ}$, but the number of such events is small.

2. We have found that for the disturbed SW types CIR, Sheath, and Rare arising from the interaction of streams with different velocities, as well as for the MC type, the probability of observing large $\left(>5^{\circ}\right)$ deviations from the radial direction is $2-5$ times higher for the latitude angle and 2-13 times higher for the longitude angle as compared to HCS, Slow, Fast, and Ejecta.

3 . We have shown that the standard deviations $\operatorname{SD}(\theta)$ and $\operatorname{SD}(\varphi)$, reflecting the level of variations in the latitude and longitude angles, respectively, have similar distributions. We determined that $\operatorname{SD}(\theta)$ and $\operatorname{SD}(\varphi)$ for CIR, Sheath, and Fast are, on average, 1.5 times greater; and for Rare, $\sim 2.5$ times greater than for HCS, Slow, $\mathrm{MC}$, and Ejecta. The probability of large $\left(>2^{\circ}\right)$ deviations from the radial direction also increases significantly for CIR, Sheath, Fast (2-8 times), and Rare (5-18 times). We demonstrated that with a decrease in the scale under study, the deviations decrease ( 4 times), and the dependence on the SW type becomes weaker (except for Rare), hence the level of local variations in the flow direction is less dependent on the large-scale stream type.

4. We have found out that Rare clearly stands out against the background of other large-scale SW stream types. Rare has the largest averages of angles and their standard deviations, always reveals wide distributions, and deviates from the radial direction much more often than other SW types.

5. The maximum of the distribution over the longitude angle $\varphi$ shifts toward negative values of $\sim-2^{\circ}$. As the proton temperature $T_{\mathrm{p}}$ and velocity $V_{\mathrm{p}}$ increase, the distribution maximum shifts to $0^{\circ}$, the distribution be- comes asymmetric, and the probability of $\varphi \geq 0^{\circ}$ increases. No dependences were found in the distributions of $\varphi$ on the magnetic field magnitude $|B|$ and the proton density $N_{\mathrm{p}}$.

6. The latitude angle $\theta$ distribution does not depend on SW parameters, and its maximum is shifted toward positive values of $\sim 1^{\circ}$.

7. Maxima of the $\operatorname{SD}(\theta)$ and $\operatorname{SD}(\varphi)$ distributions are $0.2^{\circ}-1.0^{\circ}$ for slow variations for $3600 \mathrm{~s}$ intervals and $0^{\circ}-0.4^{\circ}$ for fast variations for $30 \mathrm{~s}$ intervals. In this case, the distributions are quite wide, and the probability of occurrence of large $\operatorname{SD}(\theta)$ and $\operatorname{SD}(\varphi)$ (at intervals of 30 $\mathrm{s}>0.05^{\circ}$ ) increases with $T_{\mathrm{p}}$ from $5-10 \mathrm{eV}$ values and with $V_{\mathrm{p}}$ from $400-500 \mathrm{~km} / \mathrm{s}$ values. There is also a weak dependence of the position of the maximum on the velocity in the range $320-420 \mathrm{~km} / \mathrm{s}$ and on the temperature in the range $2-7 \mathrm{eV}$.

We are grateful to the developers of the WIND spacecraft databases [http://cdaweb.gsfc.nasa.gov] and the catalog of large-scale solar wind types [http://www.iki.rssi.ru/omni/catalog] for providing access to the data used in this study.

\section{REFERENCES}

Borovsky J.E. The flux-tube texture of the solar wind: Strands of the magnetic carpet at 1 AU? J. Geophys. Res. 2008, vol. 113, A08110. DOI: 10.1029/2007JA012684.

Borovsky J.E. The spatial structure of the oncoming solar wind at Earth and the shortcomings of a solar-wind monitor at L1. J. Atmos. Solar-Terr. Phys. 2018, vol. 177, pp. 2-11. DOI: 10.1016/j.jastp.2017.03.014.

Gosling J.T., Pizzo V.J. Formation and evolution of corotating interaction regions and their three dimensional structure. Corotating Interaction Regions. Springer, Dordrecht, 1999, pp. 21-52. DOI: 10.1007/978-94-017-1179-1_3. 
Lepping R.P., Acuna M.H., Burlaga L.F., et al. The WIND magnetic field investigation. Space Sci. Rev. 1995, vol. 71, p. 207. DOI: $10.1007 / \mathrm{BF} 00751330$.

Lin R.P., Anderson K.A., Ashford S., et al. A threedimensional plasma and energetic particle investigation for the wind spacecraft. Space Sci. Rev. 1995, vol. 71, pp. 125-153. DOI: $10.1007 / \mathrm{BF} 00751328$.

Lopez R.E. Solar cycle invariance in solar wind proton temperature relationships. J. Geophys. Res. 1987, vol. 92, p. 11189.

Yermolaev Yu.I., Nikolaeva N.S., Lodkina I.G., Yermolaev M.Yu. Catalog of large-scale solar wind phenomena during 1976-2000. Cosmic Res. 2009, vol. 47, no. 2, pp. 81-94 DOI: 10.1134/S0010952509020014.

Yermolaev Yu.I., Lodkina I.G., Nikolaeva N.S., Yermolaev M.Yu. Dynamics of large-scale solar wind streams obtained by the double superposed epoch analysis. J. Geophys. Res.: Space Phys. 2015, vol. 120. DOI: 10.1002/2015JA021274.

Yermolaev Y.I., Lodkina I.G., Yermolaev M.Y. Dynamics of large-scale solar-wind streams obtained by the double superposed epoch analysis: 3 . Deflection of the velocity vector. Solar Phys. 2018a, vol. 293, 91. DOI: 10.1007/s11207-018-1310-9.
Yermolaev Yu I., Lodkina I.G., Yermolaev M.Yu, Riazantseva M.O., Rakhmanova L.S. Statistic study of the geoeffectiveness of compression regions CIRs and Sheaths. $J$. Atmos. Solar-Terr. Phys. 2018b, vol. 180, pp. 52-59. DOI: 10.1016/ j.jastp.2018.01.027.

Zastenker G.N., Khrapchenkov V.V., Koloskova I.V., et al. Rapid variations of the value and direction of the solar wind ion flux. Cosmic Res. 2015, vol. 53, no. 1, pp. 59-69. DOI: 10.1134/ S0010952515010098.

URL: http://cdaweb.gsfc.nasa.gov (accessed October 15, 2021).

URL: http://www.iki.rssi.ru/omni/catalog (accessed October 15,2021$)$.

\section{How to cite this article}

Moskaleva A.V., Riazantseva M.O., Yermolaev Yu.I., Lodkina I.G. Variations of flow direction in solar wind streams of different types. SolarTerrestrial Physics. 2021. Vol. 7. Iss. 4. P. 10-17. DOI: 10.12737/stp74202102 . 